\title{
Modeling and kinematic simulation of the telescopic sleeve anti-swing device based on Adams depend on crane rotating
}

\author{
Guangdong Han, Haiquan Chen, Wenhua Li, Shanghai Wang \\ Marine Engineering College, Dalian Maritime University, Dalian China
}

\begin{abstract}
For offshore crane in the operation process, due to the suspension of lifting wire rope belongs to the flexible parts and wind, wave and ocean environment. These cause the load swing. A new type of offshore crane telescopic sleeve anti-swing device was proposed. It uses the casing of the rigid constraints to reduce the swing of the load. The telescopic sleeve device was introduced, and the mathematical model was established. The kinematic simulation was carried out by Adams. From the simulation result, we can get the conclusions that the telescopic sleeve has obvious effects of anti-swing.
\end{abstract}

KEYWORD: Offshore crane; Telescopic sleeve device; Modeling; Kinematic simulation; Adams

\section{INTRODUCTION}

Crane refers to a certain range of vertical lifting and horizontal movement of heavy lifting the load, and belongs to the material handling machinery. Generally, the hoisting machinery is made up of a hoisting (which enables the load up and down movement), the luffing (to make the lifting boom up and down), and the rotary mechanism (to move the load). Together with the pedestal, power plant, control and necessary auxiliary device. A working cycle includes: the crane lifts load moved to the designated place to drop down the load, and then carry out reverse motion, so that the device to return to the original place, in order to carry out the next cycle. Generally, crane through the wire rope and hook lifting load, and luffing mechanism and slewing mechanism to transfer the load. Due to the flexible parts of wire rope with luffing and rotating motion, load will make a pendulum movement, which will increase the difficulties of task and the time of working cycle. Especially for shipboard crane, due to the influence of marine environment factors, such as wind, wave and current, the ship will yawing, pitching and swaying, surging, rolling, heaving motion in six degrees of freedom, which will cause the load have a large swing. When the sea conditions is bad the crane almost impossible to work.

The mechanical and intelligent anti-swing methods are used for the crane lifting. Mechanical method is used to the crane with Maryland Rigging System ${ }^{[1]}$. The linear dynamic model of the shipboard crane is established by using Lagrange method, and application of model predictive control ${ }^{[2]}$. Optimal control method ${ }^{[1]}$ realize the effective control of the swaying of the crane. PARKER and others ${ }^{[3]}$ use RBTS (Rider Block Tagline System) to control the swing of load.

RBTS belongs to mechanical method. Its construction and control method is easier compared with Maryland Rigging System. But its function is limited when the rope is longer.

Yang Wang and others ${ }^{[4]}$ analysis the swaying of the crane, and put forward an anti-swing device of a rigid link.

In intelligent anti-swing, the Sandia national laboratory uses the POS/MV320 TSS navigation system to measure the position of the ship. The inertial measurement unit IMU, gyroscope and GPS are used to measure the movement of the ship ${ }^{[5]}$. The dynamic model of the container crane is established by JANG ${ }^{[6]}$, and the T-S fuzzy control method is adopted to control the swing of the crane. Ismail [7] establishes a three egree freedom model of the shipboard rotary crane is established. The two order sliding mode control is used to realize the trajectory tracking and swing suppression of the boom, and the impact of the structure is reduced. Pengcheng Wang ${ }^{[8]}$ modeling of the rotary crane and dynamic analysis. Yongchun Fang ${ }^{[9]}$ based on this model, they designed the nonlinear controller by Lyapunov method, the stability of the closed-loop control system is proved, and the simulation and experimental verification.

Combining with the technology status at home and abroad and the actual situation this paper pro- 
posed a telescopic sleeve anti-swing device, this paper introduces the principle of crane telescopic sleeve device for shipboard crane, and establish the mathematics model and do kinematic simulation by Adams. The simulation results indicate that the telescopic sleeve device has an obvious effects of antiswing.

\section{STRUCTURE OF TELESCOPIC CASING DEVICE}

The telescopic casing device has a universal torque structure and a telescopic sleeve. The universal torque structure is fixed on the top of the crane, and the bolt rotates, the telescopic casing and the top of the universal torque structure are connected through the screw. The inner tube and the outer tube of the telescopic sleeve are connected with the strengthening plate. The upper half part is the universal torque mechanism, the lower part is the telescopic casing. The upper part of the universal torque mechanism is composed of a hanging ear, a tube body, a damper, a ball hinge. The half part of the telescopic sleeve is made up of the outer tube, the inner pipe and the end cover. The two part is connected with the strengthening board. The retractable wire rope crane can be telescopic tube drive.

\section{ANTI-ROLLING PRINCIPLE OF TELESCOPIC CASING DEVICE}

The anti-rolling principle of the telescopic casing device is shown in Figure 1. When the crane boom is raised, the telescopic adjustment of the hydraulic cylinder, the top of the cylinder body around the fulcrum (hanging ears and lifting the top end of the hinge), so that the cylinder is always in a vertical downward state. When lifting heavy loads, because of the wave caused by the ship's rolling and pitching, resulting in a crane to produce a rolling and pitching, so that the load swing. At this point, because the wire rope through the telescopic casing device, so that the universal joint torque mechanism connected to the telescopic casing will withstand a certain wave load, and passed to the joint of the ball joint. At this point, the joint action of the three dampers connected with the ball hinge is offset by the torque of the telescopic casing passing through the ball joint, and the load is reduced. So as to achieve the purpose of anti-roll.

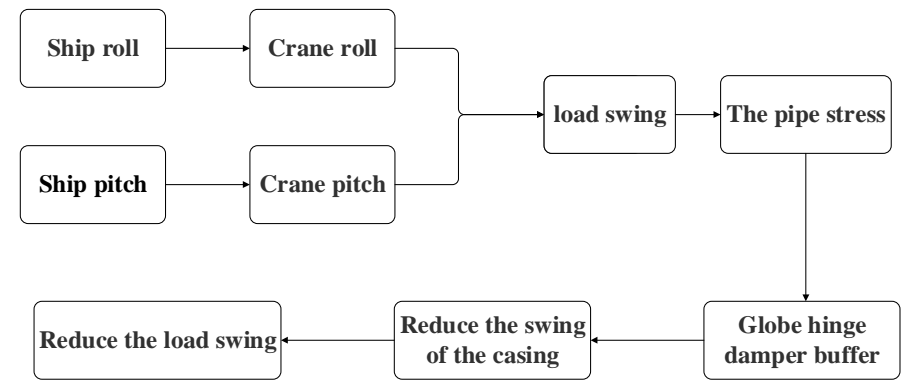

Fig.1 The anti-rolling principle of the telescopic casing device

\section{MATHEMATICS MODELING}

In order to achieve the purpose of reducing the sway of the telescopic sleeve, firstly we establish the kinematic model of the telescopic sleeve, and use the Denavit-Hartenberg method to establish the model of system. The model of the ship crane and the balance torque model of the telescopic sleeve.

\subsection{Ship crane model}

Ship crane lifting diagram model as shown in Figure2. The corresponding connecting rod parameters are shown in Table 1 . Where $\{B\}$ is the ship coordinate system, $\mathrm{Z}_{\mathrm{B}}$ is in vertical direction, $\mathrm{x}_{\mathrm{B}}$ is beam direction, $\{0\}$ for crane base coordinate system, $\{1\}$, $\{2\}$ are corresponding to the crane rotary joint, variable amplitude joint, $\theta_{1}$ and $\theta_{2}$ respect the two joints, The origin of the $\{2\}$ corresponds to the crane lifting point, $L_{1}$ is the height of basic, $L_{2}$ is the length of crane boom.

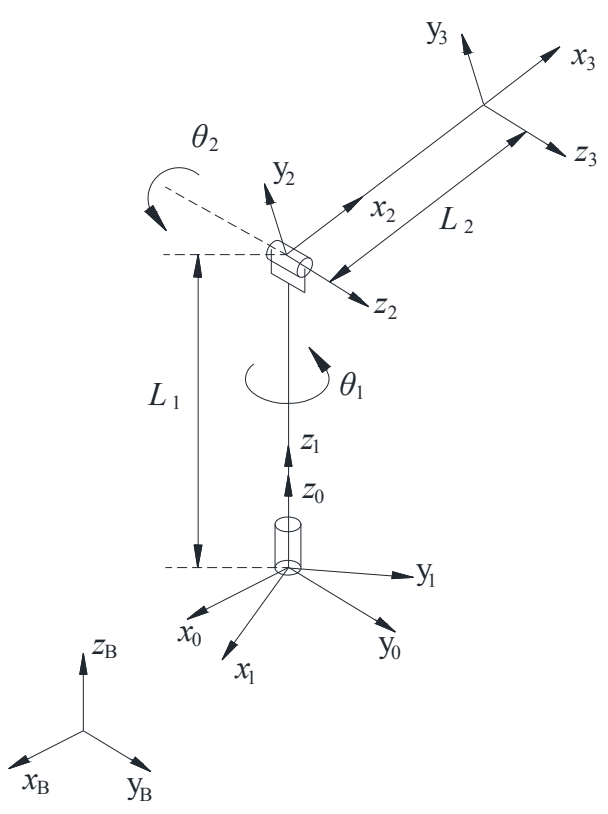

Fig. 2 Ship crane lifting diagram model 


\begin{tabular}{lllll}
\hline$i$ & $\alpha_{\mathrm{i}-1}$ & $a_{\mathrm{i}-1}$ & $d_{\mathrm{i}}$ & $\theta_{\mathrm{i}}$ \\
\hline 1 & 0 & 0 & 0 & $\theta_{1}$ \\
2 & -90 & 0 & $L_{1}$ & $\theta_{2}$ \\
3 & 0 & $L_{2}$ & 0 & 0 \\
\hline
\end{tabular}

Coordinate $\{i\}$ relative to the coordinate $\{i-1\}$.

$$
{ }_{i}^{i-1} T=\left[\begin{array}{cccc}
c \theta_{i} & -s \theta_{i} & 0 & a_{i-1} \\
s \theta_{i} c \alpha_{i-1} & c \theta_{i} c \alpha_{i-1} & -s \alpha_{i-1} & -s \alpha_{i-1} d_{i} \\
s \theta_{i} s \alpha_{i-1} & c \theta_{i} s \alpha_{i-1} & c \alpha_{i-1} & c \alpha_{i-1} d_{i} \\
0 & 0 & 0 & 1
\end{array}\right]
$$

In Eq. $1, \mathrm{c} \theta_{\mathrm{i}}$ represents $\cos \theta_{\mathrm{i}}, \mathrm{s} \theta_{\mathrm{i}}$ represents $\sin \theta_{\mathrm{i}}$, and other similar expressions are used in this kind of abbreviation.

The transformation matrix of the coordinate $\{3\}$ relative to the coordinate $\{0\}$ is:

${ }_{5}^{0} T={ }_{1}^{0} T{ }_{2}^{1} T_{3}^{2} T$

The transformation matrix of the coordinate $\{0\}$ relative to the ship coordinate $\{\mathrm{B}\}$ is:

$$
{ }_{0}^{B} T=\left[\begin{array}{cccc}
1 & 0 & 0 & B_{x} \\
0 & 1 & 0 & B_{y} \\
0 & 0 & 1 & B_{z} \\
0 & 0 & 0 & 1
\end{array}\right]
$$

Where $(\mathrm{Bx}, \mathrm{By}, \mathrm{Bz})$ for the crane base coordinate $\{0\}$ origin in the coordinates of the ship coordinate $\{\mathrm{B}\}$.

If the ship is located at the static sea level, the inertial coordinate $\{B\}$ and the ship coordinate $\{N\}$. When the wind and waves flow disturbed the ship rolling and pitching, the wave motion. The transformation matrix of the coordinate $\{\mathrm{B}\}$ of the ship relative to the coordinate $\{\mathrm{N}\}$ :

${ }_{B}^{N} T=\left[\begin{array}{cccc}c \alpha c \beta & -s \alpha & c \alpha s \beta & 0 \\ c \beta s \alpha & c \alpha & s \alpha s \beta & 0 \\ -s \beta & 0 & c \beta & 0 \\ 0 & 0 & 0 & 1\end{array}\right]$

The $\alpha$ is the coordinate $\{\mathrm{B}\}$ of the ship and the inertial coordinate $\{N\}$, and the $\beta$ is the longitudinal wave angle of the ship coordinate $\{\mathrm{B}\}$ with respect to the inertial coordinate $\{\mathrm{N}\}$.

By Eq.2, Eq.3, Eq.4 the transformation matrix is the coordinate $\{5\}$ relative to the inertial coordinate $\{\mathrm{N}\}$.

$$
{ }_{3}^{\mathrm{N}} T={ }_{B}^{N} T_{0}^{B} T_{3}^{0} T=\left[\begin{array}{cccc}
r_{11} & r_{12} & r_{13} & p_{x} \\
r_{21} & r_{22} & r_{23} & p_{y} \\
r_{31} & r_{32} & r_{33} & p_{z} \\
0 & 0 & 0 & 1
\end{array}\right]
$$

We can get:

$$
\begin{aligned}
P_{x}= & B_{x} \mathrm{c} \alpha \mathrm{c} \beta-B_{y} \mathrm{~s} \alpha+B_{z} \mathrm{c} \alpha \mathrm{s} \beta-L_{1}\left(\mathrm{c} \theta_{1} \mathrm{~s} \alpha+\mathrm{c} \alpha \mathrm{c} \beta \mathrm{s} \theta_{1}\right) \\
& +L_{2}\left(\mathrm{c} \theta_{2}\left(\mathrm{c} \alpha \mathrm{c} \beta \mathrm{c} \theta_{1}-\mathrm{s} \alpha \mathrm{s} \theta_{1}\right)-\mathrm{c} \alpha \mathrm{s} \beta \mathrm{s} \theta_{2}\right) \\
P_{y}= & B_{y} \mathrm{c} \alpha+B_{x} \mathrm{c} \beta \mathrm{s} \alpha+B_{z} \mathrm{~s} \alpha \mathrm{s} \beta+L_{1}\left(\mathrm{c} \alpha \mathrm{c} \theta_{1}-\mathrm{c} \beta \mathrm{s} \alpha \mathrm{s} \theta_{1}\right) \\
& +L_{2}\left(\mathrm{c} \theta_{2}\left(\mathrm{c} \beta \mathrm{c} \theta_{1} \mathrm{~s} \alpha+\mathrm{c} \alpha \mathrm{s} \theta_{1}\right)-\mathrm{s} \alpha \mathrm{s} \beta \mathrm{s} \theta_{2}\right) \\
P_{z} & =B_{z} \mathrm{c} \beta-B_{x} \mathrm{~s} \beta+L_{1} \mathrm{~s} \beta \mathrm{s} \theta_{1}- \\
& L_{2}\left(\mathrm{c} \theta_{1} \mathrm{c} \theta_{2} \mathrm{~s} \beta+\mathrm{c} \beta \mathrm{s} \theta_{2}\right)
\end{aligned}
$$

$P_{\mathrm{x}}, P_{\mathrm{y}}, P_{\mathrm{z}}$ for the lifting point relative to the inertial coordinate $\{\mathrm{N}\}$ of the yaw, pitch, heave.

In order to realize anti-swing is expressed as

$\theta_{1} \rightarrow \alpha$

$\theta_{2} \rightarrow \beta$

\subsection{Equilibrium torque model of telescopic casing device}

Moment equilibrium model diagram as shown in Figure 3, Where $\{C\}$ is the casing coordinate system, Captain $X^{\mathrm{c}}$ is the long direction, $Y^{\mathrm{c}}$ is the beam direction, $Z^{\mathrm{c}}$ is the vertical height. $\mathrm{L}_{1}$ is the rod length between the upper end of the ball joint and the damper joint. $\mathrm{L}_{2}$ is the rod length between the upper end of the ball joint and the end of the telescopic sleeve. $\mathrm{F}$ for damper resistance, $G$ is the force of gravity, $F_{1}$, $F_{2}, F$ component for $G$ in the vertical direction and casing, the $\alpha$ is the angle between the casing and the $Z^{\mathrm{c}}$ axis, the $\beta$ is the angle between the casing and the $X^{\mathrm{c}}$ axis.



Fig.3 Moment equilibrium model diagram

In order to achieve the torque balance, the principal vector and force of the force system are equal to zero at any point. Expression of analytical expression: 
$\sum m_{x}(F)=0 ; \sum m_{y}(F)=0 ; \sum m_{z}(F)=0$

In order to, $\sum m_{x}(F)=0$ so

$F_{X 1} L_{X 1}=F_{X 2} L_{X 2}$

Because the force $F$ is provided by the buffer force and the $Z$ axis is always vertical, and the reaction force of the damper is always opposite to the casing movement direction.so:

$F_{X 2}=F \cos ^{2} \alpha \cos ^{2} \beta ; L_{X 2}=L_{2} \cos ^{2} \alpha \cos ^{2} \beta$

Because the gravity $\mathrm{G}$ is always downward, and the shear stress of the casing is always perpendicular to the casing.so:

$F_{X 1}=G \sin \alpha \cos \alpha \cos \beta ; L_{X 1}=L 1 \sin \alpha \cos \alpha \cos \beta$

To sum up can be obtained:

$F L_{2} \cos ^{2} \alpha \cos \beta=G L_{1} \sin \alpha \cos \alpha \cos \beta$

$F L_{1} \cos \alpha=G L_{2} \sin \alpha$

$F_{Z}=G \sin ^{2} \alpha=F$

In summary, in the ship roll angle $\alpha$, pitch angle $\beta$ conditions, in order to achieve anti-rolling, joint variables, meet Eq.9, the telescopic tube device by force and the buffer to meet Eq. 16.

\section{THE OVERALL DESIGN AND THE SIMULATION ANALYSIS OF PIPE DEVICE}

Complete parts in Solidworks and assemble together, then turn the model into Adams, which can overcome the disadvantages of poor ability in threedimensional modeling of Adams. Use Solidworks establish each part, including the swaying platform, cranes, telescopic sleeve parts, et, and then create a component in Solidworks, put them together and save with Parasolid format. Open saved file in Adams, use the Modify option to change the part name, set the every parts in the assembly into a rigid body, after the completion of the set to create each joint motion pair, after driving diagram as show in Fig.4.

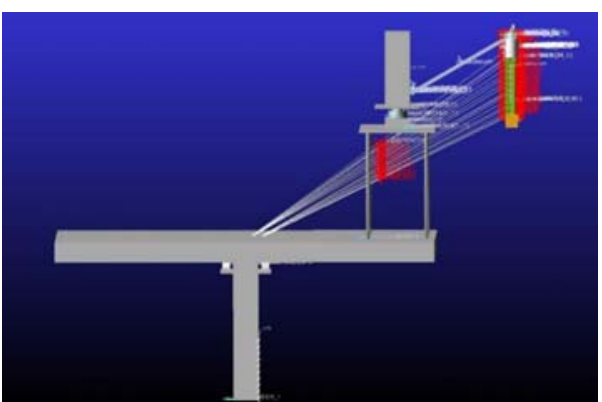

a. with telescopic sleeve

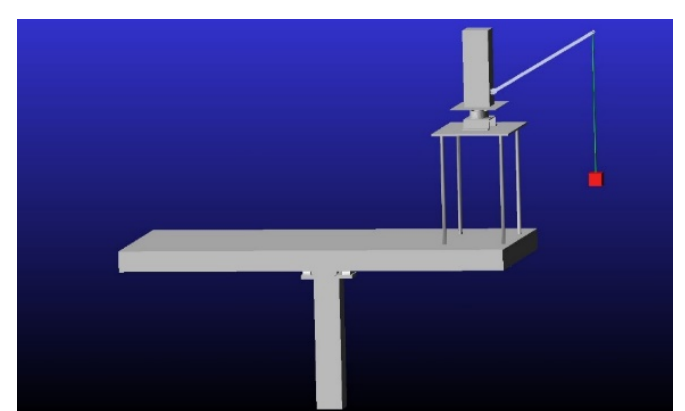

b.without telescopic sleeve

Fig.4 Modeling in Adams

Under the condition of the crane rotating, the platform is not move. The length of wire rope is $62.5 \mathrm{~cm}$, $84 \mathrm{~cm}$ and $108 \mathrm{~cm}$, respectively with and without telescopic sleeve of kinematics simulation, as shown in Table 2.

Table.2 Simulation parameters

\begin{tabular}{|l|l|l|}
\hline Group & Pendulum length & With/without casing \\
\hline E1 & 62.5 & with \\
\hline E2 & 84 & with \\
\hline E3 & 108 & with \\
\hline E4 & 62.5 & without \\
\hline E5 & 84 & without \\
\hline E6 & 108 & without \\
\hline
\end{tabular}

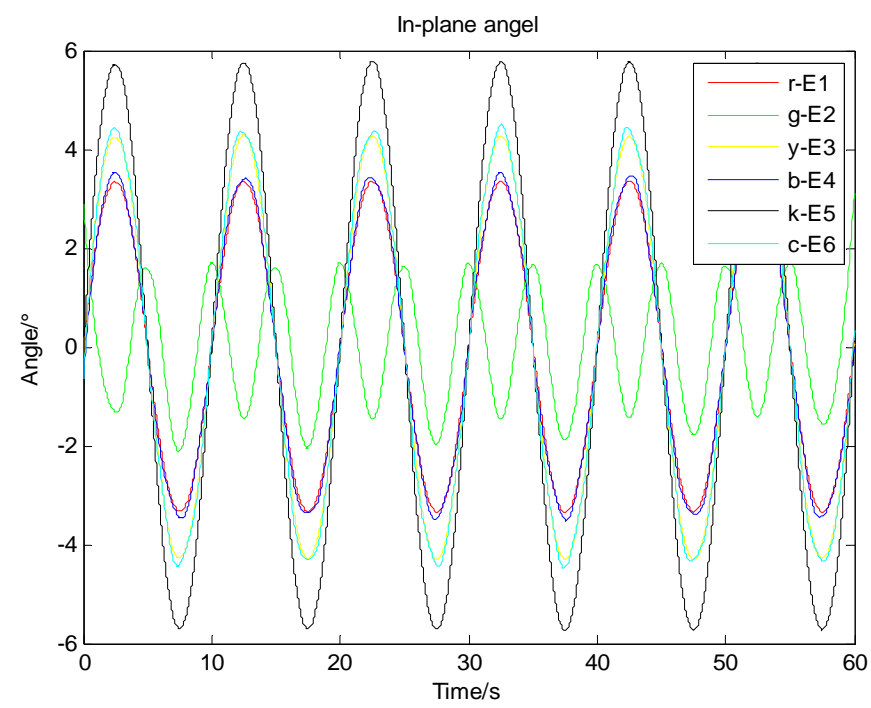

Fig.5 surface inside graph 


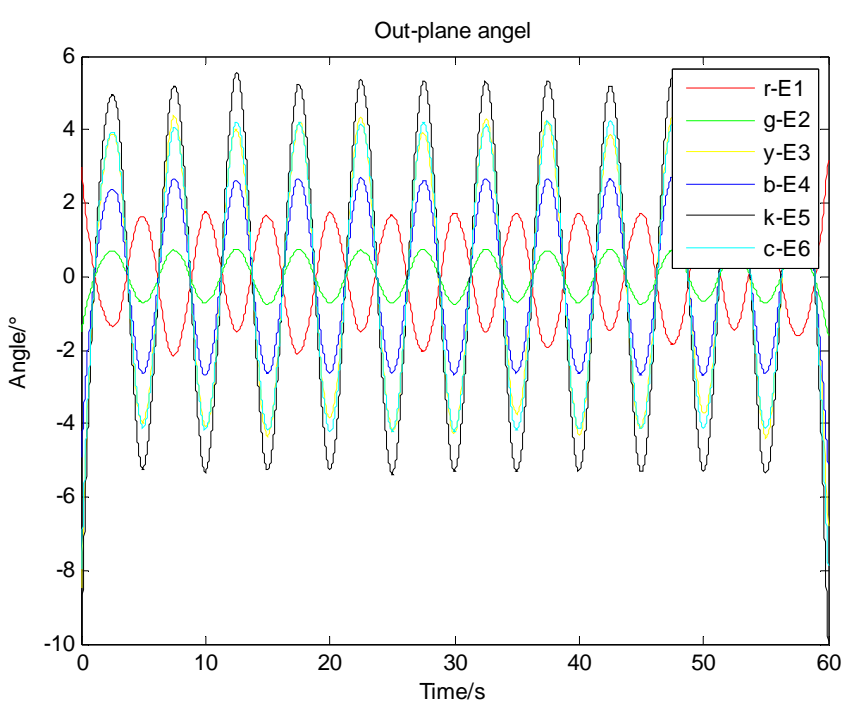

Fig. 6 surface outside graph

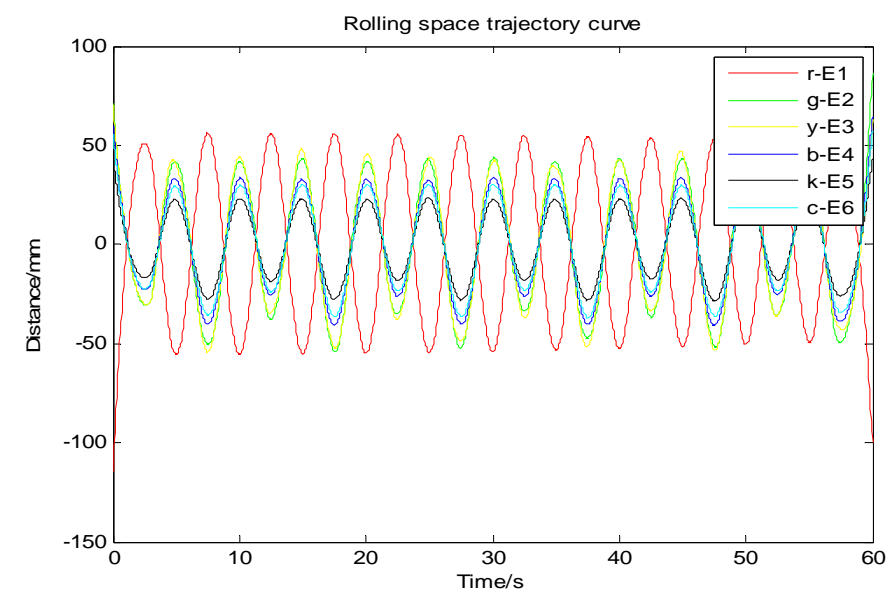

Fig.7 space trajectory curve

Fig.5, Fig.6 and Fig.7 is obtained by simulation experiment, respectively in surface inside and outside angle and space trajectory of with and without casing were analyzed. In the condition of the crane rotating, the platform is not move case. Firstly, we analysis the surface inside angle. The pendulum length is $62.5 \mathrm{~cm}$, the maximum pendulum angle is $3.2^{\circ}$ without telescopic sleeve, the maximum pendulum angle is $1.8^{\circ}$ with telescopic sleeve, relative damping amplitude is $44 \%$; The pendulum length is $84 \mathrm{~cm}$, the maximum pendulum angle is $4.4^{\circ}$ without telescopic sleeve, the maximum pendulum angle is $3.1^{\circ}$ with telescopic sleeve, relative damping amplitude is $30 \%$; Pendulum length is $108 \mathrm{~cm}$, the maximum pendulum angle is $5.7^{\circ}$ without telescopic sleeve, the maximum pendulum angle is $4.3^{\circ}$ with telescopic sleeve, relative damping rate is $25 \%$. Next, we analysis the surface outside angle. The pendulum length is $62.5 \mathrm{~cm}$, the maximum pendulum angle is $1.8^{\circ}$ without telescopic sleeve, the maximum pendulum angle is $0.6^{\circ}$ with telescopic sleeve, relative damping amplitude is $67 \%$; The pendulum length is $84 \mathrm{~cm}$, the maximum pendulum angle is $4.2^{\circ}$ without telescopic sleeve, the maximum pendulum angle is $2.5^{\circ}$ with telescopic sleeve, relative damping ampli- tude is $40 \%$; Pendulum length is $108 \mathrm{~cm}$, the maximum pendulum angle is $5.1^{\circ}$ without telescopic sleeve, the maximum pendulum angle is $3.9^{\circ}$ with telescopic sleeve, relative damping rate is $17 \%$. From the simulation results, it can be seen that the telescopic sleeve anti-swing device has an obvious anti-swing effect, about $30 \%$. From trajectory curve, the pendulum length is $62.5 \mathrm{~cm}$, the maximum pendulum distance is $33 \mathrm{~mm}$ without telescopic sleeve, the maximum pendulum distance is $22 \mathrm{~mm}$ with telescopic sleeve, relative damping amplitude is $21 \%$. The pendulum length is $84 \mathrm{~cm}$, the maximum pendulum distance is $453 \mathrm{~mm}$ without telescopic sleeve, the maximum pendulum distance is $35 \mathrm{~mm}$ with telescopic sleeve, relative damping amplitude is $22 \%$. Pendulum length is $108 \mathrm{~cm}$, the maximum pendulum distance is $53 \mathrm{~mm}$ without telescopic sleeve, the maximum pendulum distance is $45 \mathrm{~mm}$ without telescopic sleeve, relative damping rate is $18 \%$. From both with casing and without telescopic sleeve the space trajectory of change, it also proves the effect of anti-swing of the device.

\section{SUMMARY}

The mathematics model was established, also the 3D model was created by Solidworks. With two model to do statics and dynamics analysis. From the result of Kinematic simulation, we can get the telescopic sleeve anti-swing has an obvious effect of antiswing. Also verify the feasibility of telescopic sleeve stabilizer is installed.

\section{ACKNOWLEDGEMENT}

The project supported by the Special funds for basic scientific research business of the Central University (3132014345); Liaoning Natural Science Foundation of China (2015020132).

\section{REFERENCES}

[1] Wen B, Homaifar A, Bikdash M, Kimiaghalam B. Modeling and optimal control of shipboard crane[C]. American control conference, San Diego, CA, June 2-4, 1999: 593597.

[2] Kimiaghalam B, Homaifar A, Sayrrodsari B. An application of model predictive control for a shipboard crane[C]. Arlington, USA, June, 2001:929-934.

[3] Parker G, Graziano M, Leban F, et. Reducing Crane Payload Swing Using a Rider Block Tagline Control System [J]. Oceanse, Aberdeen, June, 2007:1-5.

[4] Wang Yang, Design and research of anti-sway device for marine crane Ship science and technology.

[5] Robinett, R. D., Groom, K. N., Feddema, J. T., \& Parker, G. G.. Control system and method for payload control in 
mobile platform cranes [P]. No. US 6,496,765, December 17, 2002.

[6] Jang Jae Hoon, Kwon Sung-Ha, Jeung Eun Tae. Pendulation reduction on ship -mounted container crane via T-S fuzzy model [J]. J. Cent. South Univ., 2012 (19):163-167.

[7] R. M. T. Raja Ismail, Q. P. HaTrajectory. Tracking and Anti-sway Control of Three-dimensional Offshore Boom Cranes Using Second-order Sliding Modes[C].17-20 Aug, 2013:996-1001.

[8] Yongchun Fang, Pengcheng Wang, et. Dynamic analysis and modeling of rotary swing arm crane [J] Journal of Mechanical Engineering, 2011, 47 (20):34-40.

[9] Yongchun Fang, Pengcheng Wang, Ning Sun, et al. Dynamics Analysis and Nonlinear Control of an Offshore Boom Crane [J]. 2014, 61(1):414-427. 